\title{
PROBABILISTIC FAILURE PREDICTION UNDER COMBINED IN-PLANE COMPRESSION-SHEAR LOADING FOR UNIDIRECTIONAL FIBER REINFORCED COMPOSITES
}

\author{
$\underline{\text { Nabeel Safdar }}^{1 *}$, Benedikt Daum $^{1}$, Sven Scheffler $^{1}$ and Raimund Rolfes ${ }^{1}$ \\ ${ }^{1}$ Leibniz University Hannover, Institute of Structural Analysis \\ *n.safdar@isd.uni-hannover.de
}

One of the design limiting failure for fiber reinforced composites (FRC) is under compression loads, showing failure under compression at as low as two thirds of their tensile strengths. Over the past few decades numerous analytical, experimental and numerical investigations have explored different dimensions of the problem [1]. It is now widely accepted that the dominant factors behind compression dominated failure modes are manufacturing induced fiber misalignments and the nonlinear matrix material behavior. Uncertainty regarding fiber misalignment leads to variations in strengths under compression dominated loads [2]. In order to exploit the maximum potential of FRCs, it is essential to predict the failure under axial compression and compression dominated combined loads reliably.

In order to advance the understanding of the problem in this direction, a modeling framework is presented where predictions in a probabilistic manner can be performed, leading to development of numerically calculated probabilistic failure surface under compression and combined compression-shear load cases. Figure 1 shows a periodic model schematic where the experimentally characterized fiber misalignments, shown as the contour in the figure, are also incorporated into the model. A resultant failure surface in stress space is also shown. Subsequently, this contribution presents an experimentally obtained failure surface under aforementioned load cases. The material characterization required for modeling, including fiber misalignment measurements is also performed. The differences between the modeling and experimental predictions are discussed. Finally, the numerically calculated knock down factors to account for these differences are presented.

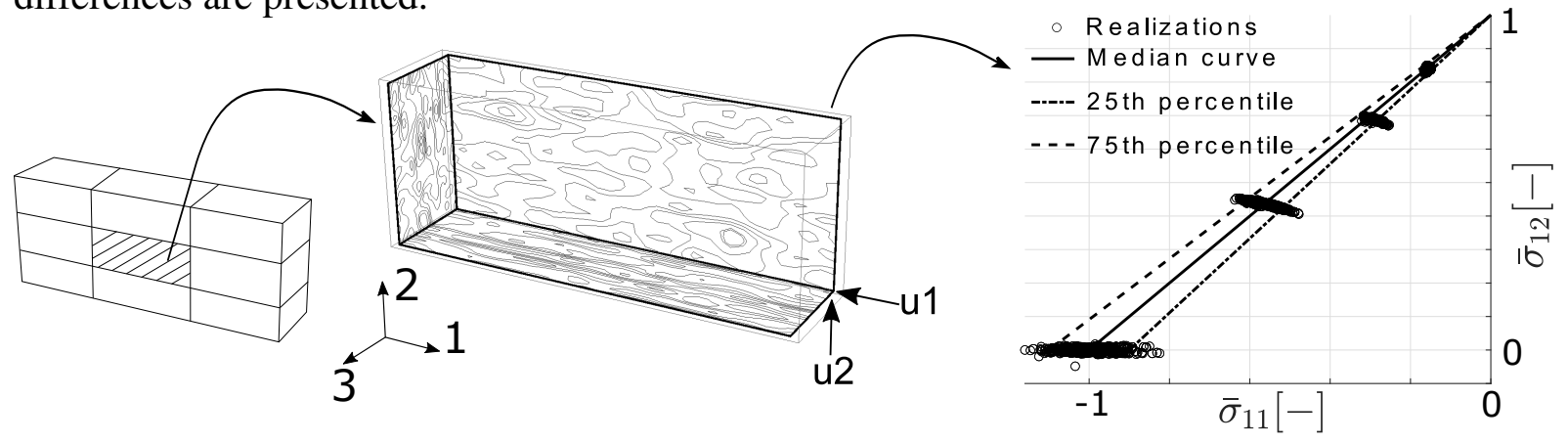

Figure 1: The figure shows modeling methodology. 1 is fiber direction whereas 2 and 3 are transversal in-plane and out-of-plane directions respectively.

\section{References}

[1] B. Daum, N. Feld, O. Allix, and R. Rolfes (2019) A review of computational modeling approaches to compressive failure in laminates. Composite Science and Technology, $\mathbf{1 8 1}$

[2] D. Liu, N.A. Fleck, and M.P.F. Sutcliffe (2004) Compressive strength of fibre composites with random fibre waviness. Journal of the Mech. and Phy. of Sol., 52(7), 1481-1505 